\title{
AX-Continuous Performance Test
}

National Cancer Institute

\section{Source}

National Cancer Institute. AX-Continuous Performance Test. NCI Thesaurus. Code C131530.

A measure of sustained attention where the subject is instructed to respond one way whenever the stimulus is an X that was preceded by an A and another way for all other stimuli, including an A, an X that was not preceded by an A, and any other letter. 